\title{
Impact of Asymmetric Traffic Densities on Delay Tolerant Vehicular Ad Hoc Networks *
}

\author{
Ashish Agarwal and Thomas D.C. Little \\ Department of Electrical and Computer Engineering \\ Boston University, Boston, Massachusetts \\ $\{$ ashisha,tdcl\}@bu.edu
}

October 21, 2009

MCL Technical Report No. 10-21-2009

\begin{abstract}
Vehicular networking can be achieved with short, medium, or long-range communication technologies. However, there are trade-offs in the adoption of these technologies including data capacity, continuity of connections, energy use and contention with other users. We focus on short range technologies that support both near-neighbor communication, for safety applications, and multihop communications for message propagation. Due to frequent network partitioning, opportunistic message exchange is required for message propagation. Earlier studies reveal that messages are suitably propagated in both directions of traffic as vehicle traffic density increases. In this paper we consider asymmetries in traffic density caused by directionality. For example, 'rush hour' traffic fills one direction of a roadway while the other direction can be sparse. Performance analysis indicates that data dissemination under asymmetry produces a corresponding asymmetry in message propagation in the direction of higher-density traffic. This result is framed in the context of traffic density regimes and is useful in the design of vehicular networks that leverage short range communications. For a fixed traffic density in one direction, an increase in density from 0 to 20 vehicles $/ \mathrm{km}$ in the other direction, yields a corresponding increase of $500 \mathrm{~m} / \mathrm{s}$ to $1000 \mathrm{~m} / \mathrm{s}$ in the messaging performance depending upon the regime.
\end{abstract}

\footnotetext{
${ }^{*}$ In Proc. 1st IEEE Vehicular Networking Conference (VNC-2009), Tokyo, Japan, October 2009. This work is supported by the NSF under grant No. and EEC-0812056. Any opinions, findings, and conclusions or recommendations expressed in this material are those of the author(s) and do not necessarily reflect the views of the National Science Foundation.
} 


\section{INTRODUCTION}

Vehicular communication is considered as an enabler for driverless cars of the future [1]. Presently, there is a strong need to enable vehicular communication for applications such as safety messaging, traffic and congestion monitoring and general purpose Internet access. Vehicular ad hoc networks (VANETs) is a term used to describe the spontaneous ad hoc network formed over vehicles moving on the roadway. Vehicular networks are fast emerging as a niche area for developing and deploying new and traditional applications. The scope and requirements of applications vary significantly, and existing techniques do not essentially apply.

Ongoing efforts are aimed at standardization of protocols and techniques to implement vehicleto-vehicle (V2V) and vehicle-to-infrastructure (V2I) communication. Challenges in enabling intervehicle communication include high mobility rates of vehicles, large topology of the network and time-varying connectivity. There are several models discussed in related work for interconnecting vehicles on the roadway. An infrastructure-based model utilizes existing or new infrastructure such as cell towers or access points (WiFi) to enable messaging.

We consider a mobile ad hoc networking model in the absence of deployed infrastructure and seek to enable data propagation for the spontaneous network formed by vehicles on a roadway setting. As any commuter knows, vehicle traffic density varies significantly with time of day and location. Due to sparsity of vehicles that can exist, end-to-end connectivity of the network is highly unlikely $[2,3]$. As a result, the network is partitioned in networking terminology. However, this partitioning is time-varying as vehicles move in opposing directions on the roadway. In fact, the network can be highly fragmented but due to mobility, has intermittent connectivity and will form many dynamic and ephemeral network configurations.

Previous work describes a routing protocol that enables networking in scenarios with varying traffic densities [4]. The technique involves attributing (labeling) messages with source-destination information so that each node in the network can make independent routing decisions based on location information derived from GPS. Application of concepts from delay tolerant networking (DTN) research [5], such as caching of messages and custody transfer mechanism, enable directional propagation of information. With the application of these concepts, the transient connectivity can be exploited to achieve successful message propagation. Results indicate via analysis and simulation that gains can be achieved by the proposed method over existing routing techniques $[6,7]$.

In this paper we consider asymmetries in traffic density caused by directionality - when densities of traffic vary from one side of the road to the other. This establishes that utilizing vehicles moving in the opposing direction is useful for data forwarding, especially in sparse density scenarios.

The remainder of this paper is organised as follows: Section 2 discusses the related work in VANET research. In Section 3, we describe our VANET routing model. Based on this model for data exchange, we describe the analytical model developed to evaluate performance of messaging, Section 4. The results obtained from this model are described in Section 5. Finally we conclude the paper in Section 6 with a summary of our results. 


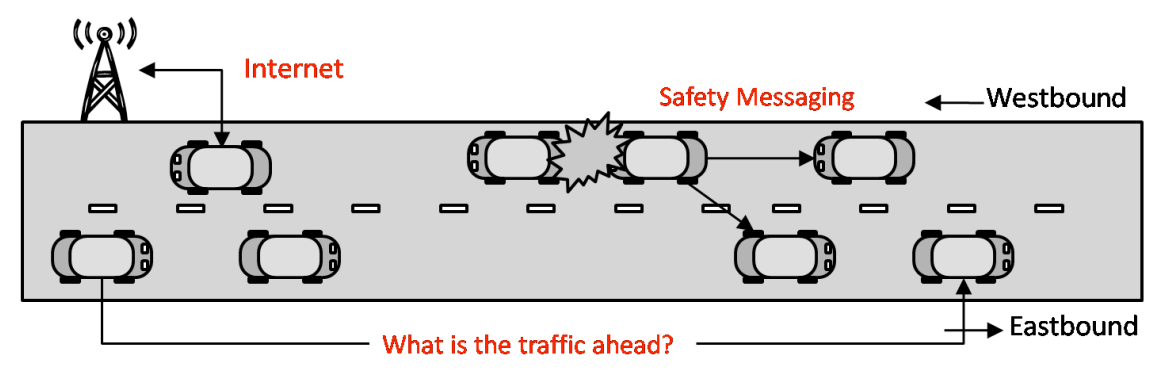

Figure 1: Illustration of data exchange in the VANET environment.

\section{RELATED WORK}

Several working groups such as PATH (The Partners for Advanced Transit and Highway), C2CC (Car-To-Car Consortium) and NOW (Networks on Wheels) [8, 9, 10] have been formed to lead efforts at improving safety and bring information services to the traveler. The initiative involves government, industrial and academic bodies. WAVE (Wireless Access for Vehicular Environments) [11] is the IEEE 802.11p draft under development to define standards and protocols to enable communication between vehicles (V2V) and between vehicles and other infrastructure (V2I).

Delay tolerant networks (DTNs) [5], also known as Intermittently Connected Mobile Networks (ICMNs) or Opportunistic Networks, are characterized by periods of connectivity interspersed with periods during which nodes are largely disconnected. Delay tolerant networking has found applications in inter-planetary space communications, mobile ad hoc networks, and sensor networks. Performance modeling in the context of ad hoc networks, particularly delay and throughput effects, is of particular interest. An important observation is the absence of end-to-end connectivity in vehicular networks owing to the unique characteristics of vehicle mobility and time-varying vehicular density. While existing mobility models such as the Freeway and Manhattan model capture the mobility of vehicles along restricted pathways, they do not adequately reflect the fragmented connectivity. However, opportunistic connectivity allows us to employ a store-carry-forward mechanism, essentially a greedy approach. In the context of vehicular networks, DTN messaging has been proposed in previous work in references [2, 4, 12, 13]. In reference [2], the authors have evaluated vehicle traces on the highway and demonstrated that they closely follow exponential distribution of nodes. The work demonstrates network fragmentation and the impact of time varying vehicular traffic density on connectivity and hence, the performance of messaging.

Several works have developed analytical models studying message propagation in VANETs. In reference [14], the authors study in detail the propagation of critical warning messages in a vehicular network. The authors develop an analytical model to compute the average delay in delivery of warning messages as a function of vehicular traffic density. Our work is unique in that we consider general purpose data propagation in the event of a partitioned network. However, our model is consistent with this work with respect to the network assumptions, e.g., exponential distribution of nodes in a one-dimensional highway setting. Another model proposed in reference [15], assumes exponential distribution of nodes to study connectivity based on queueing theory. The authors describe the effect of system parameters such as speed distribution and traffic flow to analyze the impact on connectivity. However, the authors do no consider a scenario of dynamic 
network with bidirectional mobility.

$\mathrm{Wu}$ et al. have proposed an analytical model to represent a highway-vehicle scenario [12]. They investigate speed differentials between vehicles traveling in the same direction to bridge partitioned network of vehicles. Our work is distinguished by a more complete model considering bidirectional connectivity which is intuitively faster due to the speed differential in traffic moving in opposing directions. With such a model we have demonstrated that the transient connectivity offered by opposing traffic can provide a substantial improvement in message propagation speed, beyond a certain critical threshold on traffic density.

\section{ROUTING IN THE VANET ENVIRONMENT}

VANETs (Vehicular ad hoc networks) are characterized by high mobility, rapidly changing topology, and ephemeral, one-time interactions. Adopting existing routing protocols from MANET research is challenging particularly due to an inherent MANET assumption of a relatively closed universe of interacting nodes with repeat interactions. There are several models discussed in related research for interconnecting vehicles on the roadway. An infrastructure-based model utilizes existing or new infrastructure such as cell towers or access points (WiFi) to enable messaging. Another solution is a hybrid model where vehicles communicate multihop supported by intermittently placed access points. In our work, we assume an ad hoc model, a scenario where vehicles communicate with each other, utilizing multihop networking implemented by short-range communication. There are several challenges to enabling networking in such a scenario that are discussed at length in reference [4].

Applications such as safety messaging are near-space applications where vehicles in close proximity, typically of the order of few meters, exchange status information to increase safety awareness. The aim is to enhance safety by alerting of emergency conditions. The messaging has strict latency constraints, of the order of few milliseconds, with very high reliability requirements. In contrast, applications such as traffic and congestion monitoring require collecting information from vehicles that span multiple kilometers. The latency requirements for data delivery are relatively relaxed, i.e. they are 'delay-tolerant,' however, the physical scope of data exchange is much larger. Finally, general purpose Internet access requires connectivity to the backbone network via infrastructure such as road-side access points. These are illustrated in Figure 1.

A key observation in the VANET environment is the time-varying traffic density of vehicles on the roadway. The traffic density of vehicles on the roadway varies in time, (night or day), and space. Urban areas tend to be densely populated while rural areas have sparse traffic. Thus, connectivity in the network varies between extremes of fully connected network and a sparse network with several partitions. Furthermore, it has been shown by empirical observation, [3], vehicles tend to travel in blocks that are separated from each other. Thus, in networking terms, the nodes are partitioned from each other. As a result, message propagation in the network is constrained by the occurrence of partitions between nodes. A partitioned network is illustrated in Figure 2(a).

A messaging scheme has been proposed that enables message propagation in the event of network partitioning [4]. A brief description of the scheme is provided here. The scheme relies on source and destination pairs identified on the basis of location. A common assumption in the VANET environment is GPS equipped vehicles that are location aware and share this information in a neighborhood. The location coordinates obtained from GPS are embedded in each packet such 
that each packet is attributed (labelled). Thus, we are able to implement a simplified geographic routing protocol as each intermediate node forwards data based on its location and the sourcedestination locations embedded in the data packets. The scheme does not require the formation of an end-to-end path, rather it is a connectionless messaging paradigm.

We exploit time-varying connectivity to opportunistically bridge the partitions in the network. When vehicles traveling in one direction are partitioned, vehicles that are traveling in the opposing direction are used to bridge, as illustrated in Figure 2(b). This transient connectivity can be used irrespective of the direction of data transfer, eastbound or westbound. However, it is important to note that this connectivity is not always instantaneously available. Partitions exist on either side of the roadway and in a sparse network there are large gaps between connected subnets. Here, we propose the application of delay tolerant networking (DTN) [5], [16]. We adapt techniques such as store-carry-forward mechanism and custody transfer mechanism that enable directed dissemination of data [4].

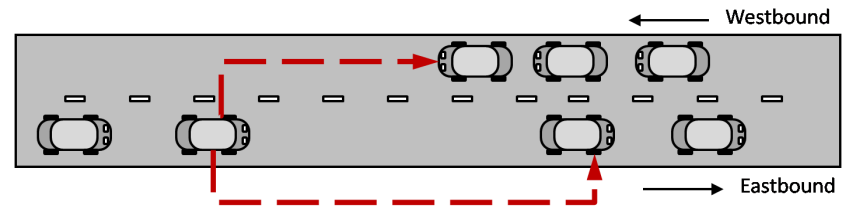

(a) At $t=0$, the network is partitioned and nodes are unable to communicate.

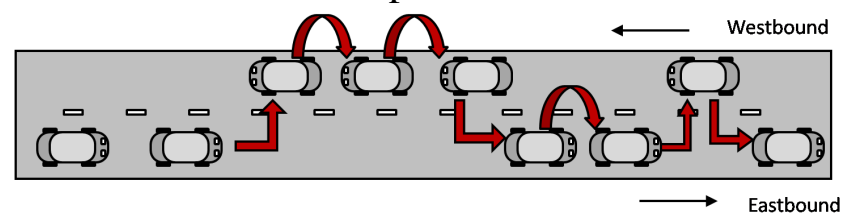

(b) At $t=\Delta t$, topology changes, connectivity is achieved and vehicles are able to communicate.

Figure 2: Illustrating delay tolerant network (DTN) messaging as the network connectivity changes with time.

Consider a message propagation goal in the eastbound direction. At time of reference $t=0$, the network is partitioned. The message originating at a vehicle encounters a partition, as shown in Figure 2(a). As the network is partitioned, the message is cached within a node's memory. By virtue of vehicle mobility, the topology of the network changes. Connectivity is sought over westbound nodes as the eastbound nodes are partitioned. For connectivity to the next eastbound node, there should be sufficient density of nodes along westbound to bridge the partition. At time $t=\Delta t$, connectivity is achieved, the messages are able to propagate multihop over connected nodes in either eastbound or westbound direction until the next partition is encountered. Thus, the message propagation alternates between periods of multihop propagation and disconnection.

\section{Analytical Model}

In this section, we briefly describe an analytical model used to derive the performance of messaging in a delay tolerant vehicular network. We build upon existing work in analytical modeling [7], [14]. Our work focuses on the scenario where there is transient connectivity in the network. The data are cached at intermediate nodes as the network is partitioned. The network alternates between periods 
of disconnection and multihop connectivity. Our aim is to compute the average data dissemination rate. We define the primary metric of interest in this paper as the average message propagation speed $\left(v_{\text {avg }}\right)$, which is similar to vehicle speed. We have described that data are spatially related and define the messaging goals in terms of location. Thus, the average message propagation speed is the rate at which messages propagate a physical distance. Without loss of generality, we focus on computing the average message propagation speed in the eastbound direction (the westbound average propagation speed can be found by simply substituting east and west indices in all the formulae).

We consider a bidirectional roadway scenario wherein vehicles travel in either direction. Vehicles are assumed to be point objects such that the length of a vehicle is not taken into account while computing distance. The model is a linear one-dimensional approximation of the roadway absent any infrastructure, such that vehicles form nodes of a linear ad hoc network. In each direction, nodes are assumed to move at a constant speed $v \mathrm{~m} / \mathrm{s}$ such that the distance between nodes moving along the same direction remains unchanged. We assume a fixed transmission range $R$. Thus, two nodes are directly connected by a radio link if the distance between them is $R$ or less. The distance $X$ between any two consecutive nodes is an i.i.d. exponential random variable, with parameter $\lambda_{e}$ for eastbound traffic and $\lambda_{w}$ for westbound traffic. The exponential distribution has been shown to be in good agreement with real vehicular traces under uncongested traffic conditions, e.g., fewer than 1,000 vehicles per hour [2].

We refer to the alternating periods of disconnection and (multihop) connectivity as phase 1 and phase 2, respectively. In phase 1 , when nodes are disconnected, by the assumption of delay tolerance, data messages are buffered at nodes until connectivity becomes available through a subset of nodes moving in the opposing direction. The messages traverse a physical distance as the vehicle travels at speed $v \mathrm{~m} / \mathrm{s}$, waiting for connectivity to be renewed. In phase 2, when multihop connectivity is available, data propagate at radio speed $\left(v_{\text {radio }}\right)$. The multihop radio propagation speed is determined by characteristics of the physical and network layers. It is typically at least an order of magnitude larger than the vehicle speed, i.e. $v_{\text {radio }}>>v$. A typical value is $v_{\text {radio }}=$ $1,000 \mathrm{~m} / \mathrm{s}$, as obtained from measurements [12]. The parameters are based on related work in short range communication implemented with 802.11 radios. However, our model is parametrized to adapt to emerging technologies. Thus, the average message propagation speed $\left(v_{\text {avg }}\right)$ is a function of the time spent in the two alternating phases.

Determining the probability that the nodes in one direction are connected through nodes traveling in the opposite direction is a difficult combinatorial problem. To circumvent this difficulty, we discretize the roadway into cells, each of size $l$. We choose an upper bound value of $l=R$ and a lower bound value of $l=R / 2$, such that the upper bound is necessary condition for connectivity but not sufficient, while the lower bound is a sufficient condition for connectivity but not always necessary. For detailed derivation of the bounds, we refer to [7]. The model is illustrated in Figure 3.

We assume the partition along eastbound direction is equivalent to $N$ cells as per the discretization. In the event that not all of the $N$ cells in the westbound direction are occupied, the nodes along eastbound are deemed to be disconnected. A message is buffered in the node's cache until connectivity is achieved. The node and, hence, the message traverse some distance (cells) until connectivity is achieved. The number of cells traversed until connectivity is analogous to the number of trials until a sequence is seen. This is described as pattern matching in classical probability theory [17]. The pattern matching problem describes the task to compute the expected number of 


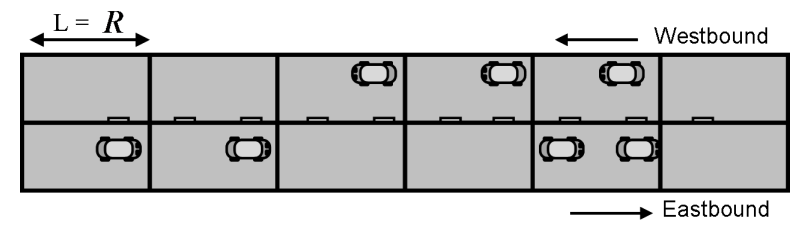

(a) Upper bound: With $l=R$, necessary but insufficient condition.

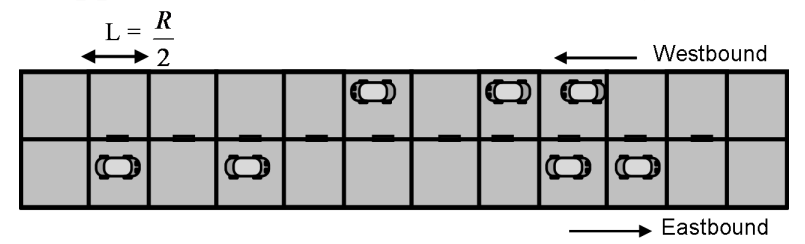

(b) Lower bound: With $l=R / 2$, sufficient but not always necessary condition.

Figure 3: Illustrating the discretization of node distribution on the roadway, upper and lower bounds for connectivity.

trials $Y$ until $N$ consecutive successes are obtained, which is given by the relation:

$$
E[Y]=\frac{1-p^{N}}{(1-p) p^{N}},
$$

where $p$ is the probability of success in a trial. This is analogous to our problem as we try to find the number of cells traversed by a node until $N$ consecutive cells along westbound traffic are occupied by one or more nodes.

Denote by $T_{1}^{n}$ and $T_{2}^{n}$ the (random) amounts of time a message spends in the two phases, during the $n$-th cycle. The random vectors $\left(T_{1}^{n}, T_{2}^{n}\right), n \geq 1$ are i.i.d., due to the memoryless assumption on the inter-vehicular distances. Note, however, that $T_{1}^{n}$ and $T_{2}^{n}$ are not independent. For instance, suppose that, at cycle $n$, the distance between the current vehicle carrying the message and the next one traveling in the same direction is larger than average, then $T_{1}^{n}$ and $T_{2}^{n}$ are more likely to be large as well.

Based on our statistical assumptions, the system can be modeled as an alternating renewal process [17], where message propagation cyclically alternates between phases 1 and 2 . Denote $E\left[T_{1}\right]=E\left[T_{1}^{n}\right]$ and $E\left[T_{2}\right]=E\left[T_{2}^{n}\right]$ as the expected time spent in phase 1 and phase 2, respectively. Then, the long-run fraction of time spent in each of these states is respectively [17]:

$$
p_{1}=\frac{E\left[T_{1}\right]}{E\left[T_{1}\right]+E\left[T_{2}\right]} ; \quad p_{2}=\frac{E\left[T_{2}\right]}{E\left[T_{1}\right]+E\left[T_{2}\right]} .
$$

Given that the average time spent in phase 1 and phase 2 are $E\left[T_{1}\right]$ and $E\left[T_{2}\right]$ respectively, while the rate of propagation in each phase is $v \mathrm{~m} / \mathrm{s}$ and $v_{\text {radio }} \mathrm{m} / \mathrm{s}$ respectively, we can compute the average message propagation speed $v_{\text {avg }}$ as follows:

$$
\begin{aligned}
v_{\text {avg }} & =p_{1} v+p_{2} v_{\text {radio }} \\
& =\frac{E\left[T_{1}\right] v+E\left[T_{2}\right] v_{\text {radio }}}{E\left[T_{1}\right]+E\left[T_{2}\right]} .
\end{aligned}
$$



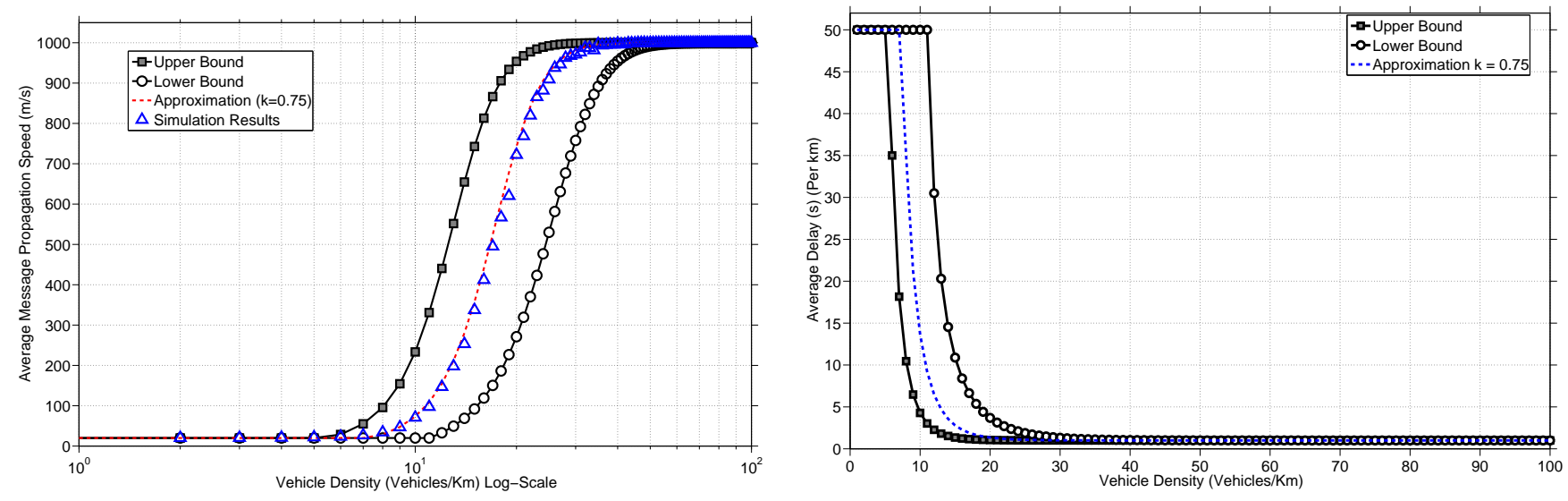

Figure 4: Performance results, for symmetric traffic densities - (a) Average message propagation speed as vehicular traffic density in the network increases. (b) Average delay, per km, as vehicular traffic density increases.

\section{PERFormance Results}

We evaluate the performance of delay tolerant messaging with the help of the analytical model. We compare the results obtained from the analytical model with those obtained from simulation. The results essentially demonstrate the performance of the messaging scheme in a network with timevarying connectivity as a function of the vehicle traffic density, transmission range and vehicle speed. The goal of analysis is to determine how $E\left[T_{1}\right]$ and $E\left[T_{2}\right]$ (and thereby the average message propagation speed $v_{\text {avg }}$ ) depend on the parameters of vehicle traffic density $\left(\lambda_{e}\right.$ and $\left.\lambda_{w}\right)$.

For comparison, we chose parameters for message propagation speed as $v_{\text {radio }}=1,000 \mathrm{~m} / \mathrm{s}$ [12]. The radio range is $R=125 \mathrm{~m}$ and the vehicle speed is assumed to be $v=20 \mathrm{~m} / \mathrm{s}$ ( $72 \mathrm{kph}$ / 45mph). The traffic density is varied from over a range of 1 vehicle $/ \mathrm{km}$ to $100 \mathrm{vehicles} / \mathrm{km}$, to cover the low, intermediate and high traffic density scenarios.

Results in Figure 4(a) depict the average message propagation speed for increasing vehicular traffic density. The traffic density is assumed to be numerically equivalent in both eastbound and westbound direction. We plot the upper bound, lower bound and the approximation results. When the mean value of vehicle traffic density is below 10 vehicles $/ \mathrm{km}$, the network is essentially disconnected and the messages are buffered within vehicles. The data traverse physical distance at vehicle speed $(v=20 \mathrm{~m} / \mathrm{s})$. When the node density is high ( $>50$ vehicles $/ \mathrm{km})$, the network is largely connected. Thus, data are able to propagate multihop through the network at the maximum speed permitted by the radio ( $v_{\text {radio }}=1,000 \mathrm{~m} / \mathrm{s}$ ). In medium node density, the network is comprised of disconnected sub-nets. There is transient connectivity in the network as vehicular traffic moves in opposing directions. As a result of the delay tolerant networking assumption and opportunistic forwarding, the message propagation alternates in the two phases. The average rate, a function of the time spent in each phase, is between the two extremes of $v \mathrm{~m} / \mathrm{s}$ and $v_{\text {radio }} \mathrm{m} / \mathrm{s}$. Thus, the message propagation speed is a function of the connectivity in the network that is in turn determined by the vehicular traffic density for constant transmission range.

Figure 4(b) shows results for average delay per kilometer in message propagation as the vehicle traffic density increases. The delay is minimum when the network is connected and messages 
propagate multihop. At lower densities, the network is disconnected and correspondingly, the delay is large. The delay, however, depends on the separation between source and destination nodes. Thus, if the separation is of several kilometers, the delay will accordingly be multiplied by a corresponding factor.

The simulation results are averaged over several iterations to account for random node generation and the resulting topology. The simulation results lie well within the upper and the lower bounds. The approximation model closely follows the simulation results. Thus, we are able to demonstrate that the analytical model captures the essence of messaging in the VANET environment characterized by time-varying connectivity and delay-tolerant networking assumption.

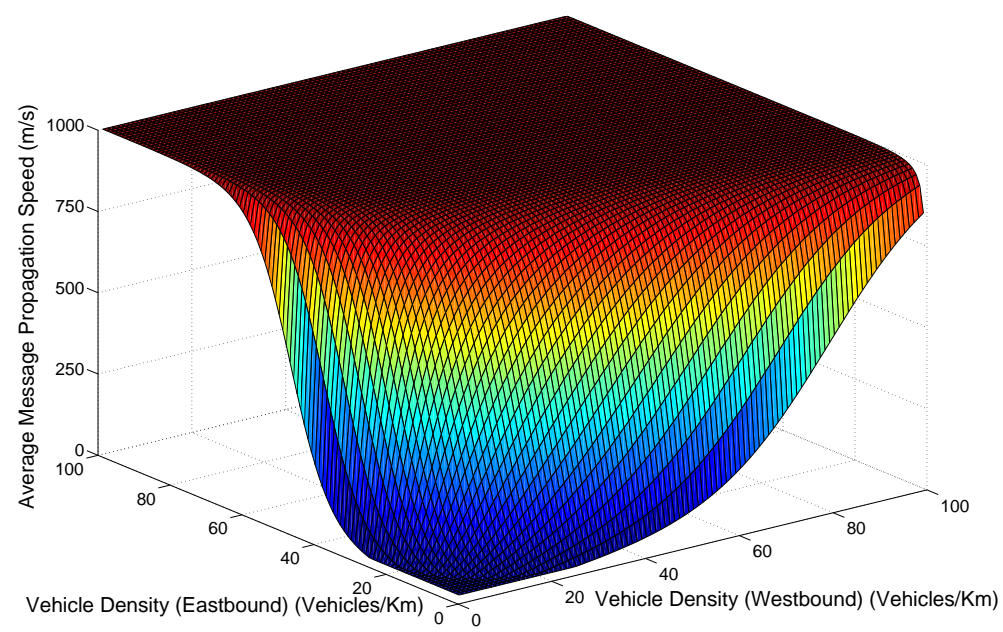

Figure 5: Average message propagation rate as a function of eastbound and westbound traffic density depicted in a 3-dimensional graph.

In Figure 5, we relax the assumption of symmetric values of traffic density along eastbound and westbound directions. We plot the graph on the basis of the approximation model for values of eastbound and westbound traffic ranging from 1 vehicle $/ \mathrm{km}$ to 100 vehicles $/ \mathrm{km}$. As is evident from the graph, the message propagation speed increases as a function of the vehicular traffic density on either side of the roadway. The 3-dimensional graph allows us to map the performance of messaging for asymmetric values of traffic density on either side of the roadway. For example, if both the eastbound and westbound directions have low traffic density of about 10 vehicles $/ \mathrm{km}$, then the node density is insufficient to enable multihop message propagation. However, if the node density in the eastbound roadway is low, say 20 vehicles/ $/ \mathrm{km}$, while the westbound direction has higher traffic density, say 40 vehicles $/ \mathrm{km}$, then the node density is sufficient to reach the maximum performance of $v_{\text {radio }}(1,000 \mathrm{~m} / \mathrm{s})$.

Figure 6(a) illustrates three different regimes of performance based on our analytical model for the case $R=125 \mathrm{~m}$. These density regimes are obtained from the minimum threshold density required in each direction of the highway to gain from the delay tolerant assumption. In Regime I, no gain is provided from the occasional opportunistic connectivity provided by the DTN architectures. In Regime III, the value of $v_{\text {avg }}$ is strictly larger than $v$ and increases with $\lambda_{e}, \lambda_{w}$ and $v_{\text {radio }}$. The figure shows that for low traffic density in one direction $(<10$ vehicles $/ \mathrm{km})$, a relatively high 

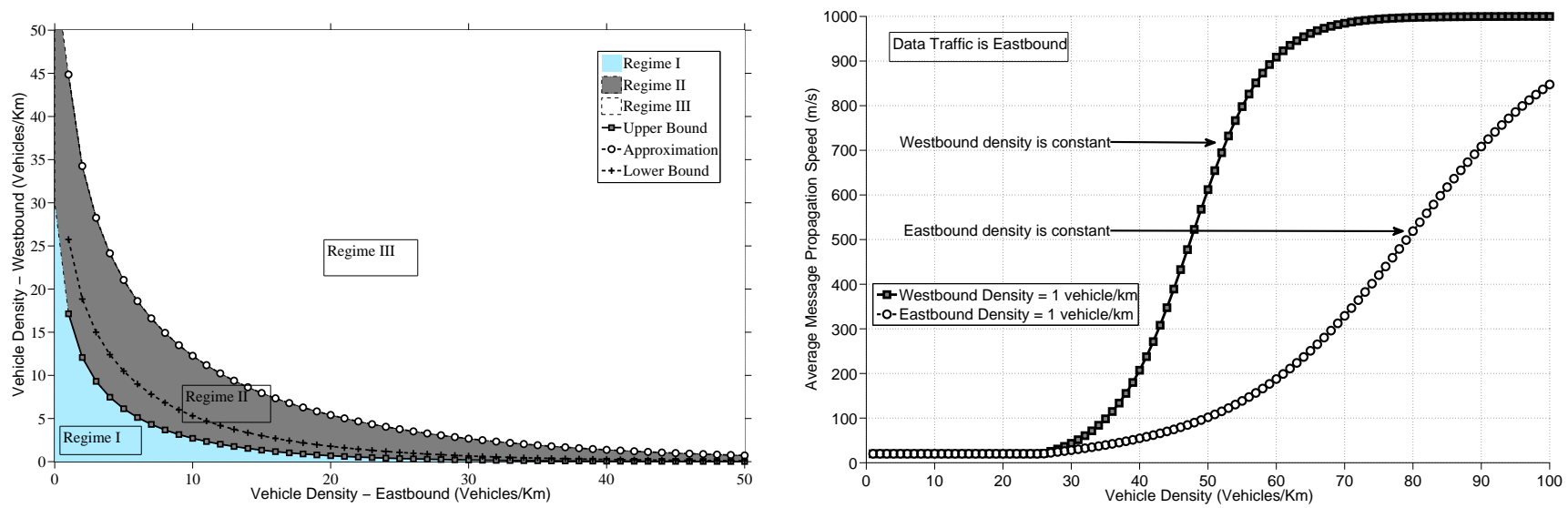

Figure 6: Performance results - (a) Density regimes for gains from the delay tolerant architecture (b) Average message propagation speed as vehicular traffic density in one direction increases; while the other direction is fixed at 1 vehicles $/ \mathrm{km}$.

density of traffic in the other direction, $(10-25$ vehicles $/ \mathrm{km})$ is required. While this result may be intuitive, the mathematical relationship is only derived from the analytical model. Regime II is the set of density values between the upper and lower bounds, where the gains are uncertain.

It is noteworthy, that in Regime I, with a small increase in traffic density in either direction, there is no significant increase in the message propagation speed, as there are no gains to be achieved by the delay tolerant architecture. However, in Regime III, a small increase in density provides immediate gains in the performance of messaging.

In Figure 6(b), we consider the scenario in which the traffic density on one side of the roadway is fixed. We compare, using our analytical model, the performance of messaging in the eastbound direction. As explained in the previous section, the alternate case can be derived by alternating the density value. For the first curve, we fix the density on the eastbound direction at 1 vehicle $/ \mathrm{km}$, and compare the messaging performance for increasing westbound density. The second curve shows the performance for fixed density in the westbound direction and increasing eastbound traffic density. This results in asymmetric traffic densities on the roadway.

Comparing performance we see that for an average of 1 vehicle $/ \mathrm{km}$, in the eastbound direction, the network is partitioned. Thus, for low densities, the messaging performance is equivalent to the vehicle speed $(v \mathrm{~m} / \mathrm{s})$. From previous analysis (Figure 6(a)), there are no gains achieved from the delay tolerant assumption until the traffic density in the westbound direction is at least 27 vehicles/km, on average. As the westbound traffic density increases the messaging speed increases. However, the maximum performance is not achieved because of network partitioning and lack of end-to-end connectivity. When we consider the westbound density to be fixed at 1 vehicle/km and the eastbound density increases, the performance characterization is different. Here, we observe that as the eastbound density increases, the partitions are smaller in size, on average, and less frequent. Thus, even a sparse network density in the westbound direction can provide significant gains.

In Figure 7(a), we compare the performance of messaging for a fixed traffic density of 15 vehicles $/ \mathrm{km}$. Again, the performance is equivalent to vehicles speed $(v \mathrm{~m} / \mathrm{s})$ until the minimum threshold density of $2-3$ vehicles $/ \mathrm{km}$ is achieved in the other direction, which exploits the transient 

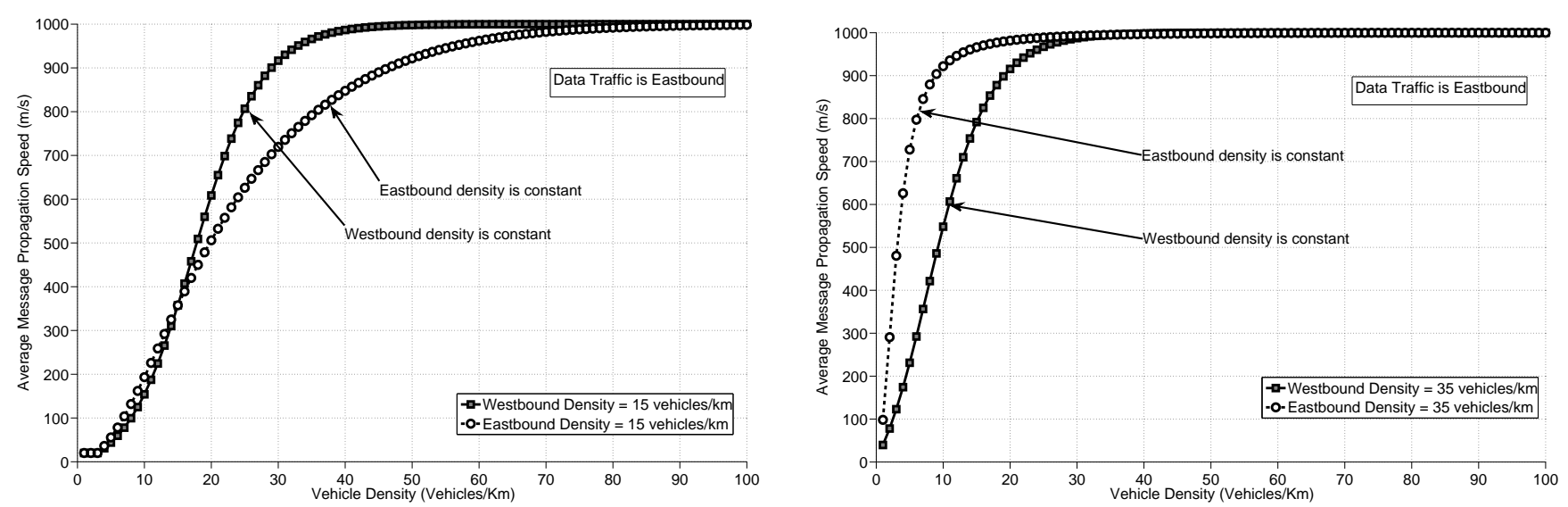

Figure 7: Performance results - Average message propagation speed as vehicular traffic density in one direction increases; while the other direction is fixed at (a) 15 vehicles $/ \mathrm{km}$ (b) 35 vehicles/km

connectivity. For fixed traffic density in the eastbound direction, once the traffic density increases beyond the minimum threshold, the messaging performance increases rapidly with traffic density. However, since the partitions in the network still exist at the same rate, the messaging performance is dependant upon the westbound traffic for connectivity. In the inverse case, when the westbound density is fixed at 15 vehicles/km and the eastbound density is increased, the size of the partitions decreases and they become less frequent. Thus, the messaging performance is dominated by eastbound density. The two curves cross each other at 15 vehicles $/ \mathrm{km}$ on the $\mathrm{x}$-axis of the graph, which occurs when the value of eastbound density exceeds the fixed density of the other curve.

Finally, Figure 7(b) shows a comparison of performance at a fixed density of 35 vehicles $/ \mathrm{km}$. Here, since the densities are higher, the partitions are smaller and infrequent. The gains achieved in messaging performance for increasing node density are therefore, significantly high. Thus, the curve increases rapidly and reaches the maximum performance value, as early as 20 vehicles $/ \mathrm{km}$ for traffic in the opposing direction. In contrast to previous graphs, the fixed density of eastbound traffic of 35 vehicles $/ \mathrm{km}$ is higher than the westbound density and dominates in the messaging performance.

Comparing the gains in messaging performance for fixed eastbound density in Figure 6(b), a small change in density in the westbound direction provides no gains until the threshold value of 27 vehicles/km. While from Figures 7(a) and 7(b), we observe that significant gains are achieved, as densities increase. An increase in westbound density from 0 vehicles $/ \mathrm{km}$ to 20 vehicles $/ \mathrm{km}$, increases the dissemination rate to $500 \mathrm{~m} / \mathrm{s}$ and $1,000 \mathrm{~m} / \mathrm{s}$ respectively. Comparing the difference in messaging performance, it is as high as $700 \mathrm{~m} / \mathrm{s}$ when the density in the other direction is 60 vehicles/km, Figure 6(b). However, for a fixed traffic density of 15 vehicles/km, the performance difference between the two curves is lower at $200 \mathrm{~m} / \mathrm{s}$, when the density in the other direction is 30 vehicles/km, Figure 7(a).

Thus, from these results we demonstrate two important insights. First, when the traffic density in the eastbound direction is low, the network is largely disconnected, the transient connectivity offered by traffic in westbound direction can be utilized for data forwarding. This supports our claim to use the traffic in both directions of the roadway for message propagation. Second, the data propagation is directional and dependant upon the traffic density in the same direction. Traffic in 
opposing direction can bridge partitions and provide intermittent connectivity, however, in sparse networks, the partitions dominate.

\section{CONCLUSION AND Discussion}

In this paper we analyzed the impact of asymmetric directional traffic densities on the performance of messaging under an opportunistic delay-tolerant messaging scheme. Results establish that the rate of directional data dissemination is a function of traffic density of the same direction. Furthermore, data dissemination rates in either direction are not always equal for asymmetric vehicular traffic. Traffic in the opposing direction can be utilized to achieve gains in performance. For a fixed traffic density, a gain in density from 0 to 20 vehicles $/ \mathrm{km}$, a corresponding increase of $500 \mathrm{~m} / \mathrm{s}$ and $1000 \mathrm{~m} / \mathrm{s}$ is achieved in the messaging performance. However, for sparse networks, the partitions in the direction of data dissemination dominate. Finally, we demonstrate the density regimes in which significant performance gains can be achieved. A density combination of eastbound and westbound traffic of 1 vehicle $/ \mathrm{km}$ and 10 vehicles $/ \mathrm{km}$, respectively, yields no gains from delay tolerant networking. In contrast, for a combination of 15 vehicles $/ \mathrm{km}$ and 20 vehicles $/ \mathrm{km}$ eastbound-westbound, signficant gains are achieved through the delay tolerant asumption.

\section{ACKNOWLEDGMENT}

The authors would like to thank Prof. David Starobinski at Boston University for prior work leading to this publication.

\section{References}

[1] USP researchers say future cars will communicate to avoid collisions. [Online]. Available: http://www.usp.ac.fj/news/story.php?id=416

[2] N. Wisitpongphan, F. Bai, P. Mudalige, and O. Tonguz, "On the Routing Problem in Disconnected Vehicular Ad-hoc Networks," INFOCOM 2007. 26th IEEE International Conference on Computer Communications. IEEE, pp. 2291-2295, May 2007.

[3] V. Naumov, R. Baumann, and T. Gross, "An Evaluation of Inter-Vehicle Ad Hoc Networks Based on Realistic Vehicular Traces," in Proc. 7th ACM Intl. Symp. on Mobile Ad Hoc Networking and Computing (MobiHoc '06), Florence, Italy, May 2006, pp. 108-119.

[4] T. D. C. Little and A. Agarwal, "An Information Propagation Scheme for Vehicular Networks," in Proc. IEEE Intelligent Transportation Systems Conference (ITSC), Vienna, Austria, September 2005.

[5] K. Fall, "A Delay-Tolerant Network Architecture for Challenged Internets," in Proc. Special Interest Group on Data Communications (SIGCOMM '03), Karlsruhe, Germany, August 2003, pp. 27-34. 
[6] A. Agarwal, D. Starobinski, and T. D. C. Little, "Exploiting Downstream Mobility to Achieve Fast Upstream Propagation," in Proc. of Mobile Networking for Vehicular Environments (MOVE) at IEEE INFOCOM 2007, Anchorage, AK, May 2007.

[7] _ _ "Analytical Model for Message Propagation in Delay Tolerant Vehicular Ad Hoc Networks," in Vehicular Technology Conference (VTC-Spring '08), Singapore, May 2008, pp. 3067-3071.

[8] Network on Wheels. [Online]. Available: http://www.network-on-wheels.de/about.html

[9] Car 2 Car Communication Consortium. [Online]. Available: http://www.car-2-car.org/

[10] The Partners for Advanced Transit and Highway (PATH). [Online]. Available: http: //www.path.berkeley.edu/

[11] I. Berger, "Standards for Car Talk," The Institute, March 2007.

[12] H. Wu, R. Fujimoto, and G. Riley, "Analytical Models for Information Propagation in Vehicle-to-Vehicle Networks," in Proc. 60th IEEE Vehicular Technology Conference (Fall VTC '04), vol. 6, Los Angeles, CA, USA, September 2004, pp. 4548-4552.

[13] T. Nadeem, P. Shankar, and L. Iftode, "A Comparative Study of Data Dissemination Models for VANETs," in Proc. 3rd Intl. Conference on Mobile and Ubiquitous Systems: Computing, Networking \& Services (MOBIQUITOUS '06), San Jose, CA, USA, July 2006, pp. 1-10.

[14] R. Fracchia and M. Meo, "Analysis and Design of Warning Delivery Service in Intervehicular Networks," IEEE Transactions on Mobile Computing, vol. 7, no. 7, pp. 832-845, 2008.

[15] S. Yousefi, E. Altman, R. El-Azouzi, and M. Fathy, "Analytical Model for Connectivity in Vehicular Ad Hoc Networks," IEEE Transactions on Vehicular Technology, vol. 57, no. 6, pp. 3341-3356, Nov. 2008.

[16] P. Jacquet, B. Mans, and G. Rodolakis, "Information propagation speed in Delay Tolerant Networks: Analytic upper bounds," IEEE International Symposium on Information Theory (ISIT 2008), pp. 6-10, July 2008.

[17] S. M. Ross, Introduction to Probability Models. Academic Press, 2004, pp. 47-52.

[18] M. M. Artimy, W. Robertson, and W. J. Phillips, "Connectivity in Inter-Vehicle Ad hoc Networks," in Canadian Conference on Electrical and Computer Engineering, 2004., vol. 1, May 2004, pp. 293-298 Vol.1.

[19] L. Briesemeister and G. Hommel, "Role-based multicast in highly mobile but sparsely connected ad hoc networks," in Proc. 1st ACM Intl. Symposium on Mobile Ad Hoc Networking \& Computing (MobiHoc 'O0). Piscataway, NJ, USA: IEEE Press, 2000, pp. 45-50.

[20] J. Burgess, B. Gallagher, D. Jensen, and B. N. Levine, "MaxProp: Routing for Vehicle-Based Disruption-Tolerant Networks," in Proc. IEEE Conference on Computer Communications (INFOCOM), Barcelona, Spain, April 2006, pp. 1-11. 
[21] K. Fall, S. Madden, and W. Hong. (2003, July) Custody Transfer for Reliable Delivery in Delay Tolerant Networks. URL. Intel Research. [Online]. Available: http://www.dtnrg.org/docs/papers/custody-xfer-tr.pdf

[22] C. H. Foh and B. S. Lee, "A Closed Form Network Connectivity Formula for OneDimensional MANETs," in Proc. IEEE ICC '04, vol. 6, June 2004, pp. 3739-3742.

[23] H. Füßler, M. Mauve, H. Hartenstein, D. Vollmer, and M. Käsemann, "MobiCom poster: Location Based Routing for Vehicular Ad Hoc Networks," in Proc. Intl. Conference on Mobile Computing and Networking (MOBICOM '02), vol. 7, no. 1, Atlanta, GA, USA, September 2002, pp. 47-49.

[24] A. Ghasemi and S. Nader-Esfahani, "Exact probability of Connectivity in One-Dimensional Ad Hoc Wireless Networks," IEEE Communications Letters, vol. 10, no. 4, pp. 251-253, April 2006.

[25] T. D. C. Little and A. Agarwal, "Connecting Vehicles to 'The Grid'," in Proc. NITRD National Workshop on High-Confidence Automotive Cyber-Physical Systems, Troy, MI, April 2008.

[26] S. Wang, "On the intermittence of routing paths in vehicle-formed mobile ad hoc networks on highways," in Intelligent Transportation Systems, 2004. Proceedings. The 7th International IEEE Conference on, Oct. 2004, pp. 803-809.

[27] H. Wu, R. Fujimoto, R. Guensler, and M. Hunter, "MDDV: A Mobility-Centric Data Dissemination Algorithm for Vehicular Networks," in Proc. 1st ACM Intl. Workshop on Vehicular Ad Hoc Networks (VANET), Philadelphia, PA, USA, 2004, pp. 47-56.

[28] W. Zhao, M. Ammar, and E. Zegura, "A Message Ferrying Approach for Data Delivery in Sparse Mobile Ad Hoc Networks," in MobiHoc '04: Proceedings of the 5th ACM International Symposium on Mobile Ad hoc Networking and Computing. New York, NY, USA: ACM, 2004, pp. 187-198.

[29] O. Dousse, P. Thiran, and M. Hasler, "Connectivity in ad-hoc and hybrid networks," vol. 2, 2002, pp. 1079-1088 vol.2. 